\title{
HABILIDADES COMUNICATIVAS Y EL DESARROLLO DEL PENSAMIENTO CRÍTICO EN ESTUDIANTES DEL CENTRO DE ALTOS ESTUDIOS NACIONALES. $1,2,3$
}

Communicative skills and the development of critical thinking in students of the center of high national studies

Fecha de recepción: 27 de abril de 2020

Fecha de aceptación: 10 de junio de 2020

1- Tamara Tatiana Pando Ezcurra. Grado académico: Doctorado en Educación. Adscripción: Centro de Altos Estudios Nacionales. Correo electrónico: tamara-tatiana2010@hotmail.com. ORCID ID: https://orcid.org/0000-0003-0301-3440

2- Raúl Eduardo Cabrejos Burga. Grado académico: Doctor en Administración. Adscripción: Red Radar. Correo electrónico: raulcabu@hotmail.com . ORCID ID: https://orcid.org/000-0001-6553-8619

3- Yoni Wildor Nicolás Rojas. Grado académico: Doctor en Educación. Adscripción: Instituto de Educación Superior Tecnológico Privado "La Pontificia". Correo electrónico: yoninicolas@gmail.com/ ORCID ID: https://orcid.org/0000-00016493-6084 


\title{
RESUMEN
}

El estudio actual surge del objetivo de determinar la correlación entre las variables habilidades comunicativas y el desarrollo del pensamiento crítico. El pensamiento crítico concebido como proceso en el cual uno piensa por sí mismo, formula preguntas en los estudiantes de postgrado del centro de altos estudios nacionales, donde permite la indagación. El tipo de investigación es básico con un enfoque cuantitativo, el diseño empleado fue no experimental con un alcance descriptivo en la cual se trabajó con una población de 40 estudiantes; se plantearon dos instrumentos los cuales pasaron por un proceso de validez y fiabilidad, para luego ser aplicado. Los datos fueron procesados, los cuales per-

mitieron aceptar una relación positiva entre las habilidades comunicativas y el desarrollo del pensamiento crítico en los estudiantes de postgrado del centro de altos estudios nacionales.

Palabras claves: Habilidades Comunicativas, Pensamiento Crítico, Estudiantes

\begin{abstract}
The present study is based on the objective to determine the correlation between communication skills and critical thinking development variables in post-graduate students of the national high specialized studies center. The used design was a non-experimental correlational descriptive study with a quantitative focus, in which we surveyed a population of 40 students; two instruments were considered, which went through a reliability validation process, for later application. Data was processed and allowed to accept that there is a significant positive correlation between communication skills and critical thinking development in post-graduate students of the national high studies center.
\end{abstract}

Keywords: Communication Skills, Critical Thinking, Students 


\section{Introducción}

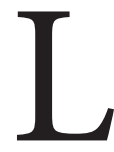

a llamada sociedad del conocimiento, en la cual nos desenvolvemos se da los cambios de forma acelerada lo cual se debe principalmente al acelerado cambio que se dan en la ciencia y la

tecnología, ello ha ido transformando la sociedad de forma significativa tanto en lo político, social y cultural; ante ello se aprecia gran cantidad de canales de comunicación que han permitido que la persona tenga acceso a la información de forma más rápida. Sin duda por más cambios acelerados que se den la comunicación sigue siendo una parte central de la vida del ser humano, pues ella les permite establecer todas las relaciones, sociales, políticas, laborales, afectivas, etc., que forman a lo largo de su existencia.

De allí la importancia de formar habilidades comunicativas sólidas, pues gran parte de los problemas tanto dentro de las organizaciones como en la vida cotidiana, están vinculadas con incompetencias para expresarse y de relacionarse con sus pares. El avance acelerado de la ciencia y la tecnología han permitido, facilitar la comunicación, y han permitido la evolución del ser humano. En el momento actual se puede observar una gran cantidad de sistemas de comunicación, acceso casi irrestricto a canales de información, bases de datos y esto, sin duda, ha cambiado los patrones de comportamiento y junto con él, las actitudes, los valores y los hábitos. Asimismo, las tecnologías han transformado el mundo laboral, de relaciones y también la educación, y en todos sus niveles. Ante ello la persona debe ser capaz de discernir ante todo lo que está a su alrededor, por lo cual debe ser capaz de utilizar un pensamiento crítico, lo cual implica que puede pensar por sí mismo, que no acepta las ideas de los demás si no está convencido de ellas.

Es decir, es capaz de evaluar las ideas y opiniones de las otras personas, poniendo siempre los pros y contras en una balanza, de manera tal que pueda tener su propia opinión, aun si esto está en contra de lo que dicen los demás o la propia sociedad. La persona debe tener sus propias valoraciones de los sucesos y del propio progreso del saber. La tecnología ligada al discernimiento nos permite contar con destrezas y fomentar la creatividad que son los grandes progresos de la humanidad

\section{Marco Teórico}

Se incluye como marco teórico a la formación del Pensamiento Crítico que viene siendo materia de reflexión directa e indirecta, con el fin de buscar métodos que permitan un resultado exitoso en el desarrollo de la formación de la persona, que repercute en su capacidad de querer mejorar la sociedad y así mismo como una forma de autorregularse; es en ese sentido que se aprecia una relación entre la formación del pensamiento crítico y la investigación (Mendoza, 2015). Este énfasis en la necesidad social del desarrollo del pensamiento crítico ha sido expresado por la Conferencia Mundial sobre la Educación Superior UNESCO (1998) y ratificado en diversas oportunidades. Se vinculó a la Investigación como elemento clave para la formación universitaria, que debe alinear los currículos con las demandas sociales, incluyendo por tanto la formación de aspectos que van más allá de la formación tradicional centrada en la adquisición de conocimientos (Cortés, 2006). 
Pensar críticamente cobra gran importancia en un momento en que el mundo, representado por los cambios y las invariables crisis que existen en todos los órdenes generales, gubernamentales y financieros que reclaman con urgencia la representación de individuos aptos para desenvolverse con sabiduría propia en la demanda de procedimientos y compromisos y la acomodación para las grandes transformaciones que se producen. Por estas razones es que Lipman (1997) orienta al estudio y desarrollo del pensamiento crítico a todas las personas responsables que certifiquen la protección de una colectividad democrática. Una sociedad comprometida es aquella que es capaz de pensar críticamente y lograr estrategias cognitivas propias del proceso reflexivo. Es preciso indicar que se pretende determinar si existe una relación entre las habilidades comunicativas y el pensamiento crítico, de esta manera, cada vez se estará más cerca de otorgar a los alumnos una educación que guarde relación con todas las competencias que el mundo moderno exige $y$, de esta manera, tener ciudadanos eficientes y altamente competitivos.

En todo este proceso la comunicación es un intercambio de información, esto encierra aspectos en todo el transcurso comunicativo de poder transmitir formas de pensar y sentir. Martínez (2002) afirma que la comunicación es un proceso por medio del cual una persona mediante mensajes se pone en contacto con otras ya sea para emitir opiniones, o brindar una respuesta; por ello la comunicación es una manera de establecer contacto a través de opiniones, percepciones y sentidos que busca una respuesta al momento de comunicarse. Para Koontz y Weihrich (2000, p. 120) la comunicación en la empresa es "el impulso que se adhiere entre sí a los sujetos y se puede llegar alcanzar un panorama favorable, alcanzando los objetivos y fines organizacionales". La comunicación es una responsabilidad compartida en la que todos los trabajadores de una institución deben actuar y hacerlo bien. Cuando se establece el término habilidades comunicativas se hace referencia a la competencia que tiene una persona para expresar sus ideas o sentimientos a través de dos maneras: el lenguaje oral y el lenguaje escrito. Así lo han expresado Monsalve, Franco, Betancur y Ramírez (2009, p. 193), quienes sostienen que "las destrezas comunicativas y las competencias sirven para que los individuos expresen sus ideas, sentimientos, necesidades, sueños y deseos por medio del lenguaje oral y escrito. De esta forma, es la capacidad que tiene el ser humano para comprender los mensajes que recibe a través de estos códigos".

En cuanto a las dimensiones de las habilidades comunicativas se señala las siguientes: La habilidad del habla, sostiene Saussure (1989) que el habla se entiende como un acto de carácter individual a través del cual se exterioriza el lenguaje. Esto permite que se puedan manifestar necesidades, pensamientos, emociones, deseos y sentimientos, así como la emisión de sonidos inherentes a una lengua que se utiliza en un espacio determinado. Para que esto se realice con precisión, es necesario que el interlocutor o destinatario maneje el mismo código lingüístico que el emisor, pues solo así se podrá interpretar los mensajes.

Cassany, Luna y Sanz (2007) señalan que en cuanto a la habilidad de escucha como la capacidad que tienen las personas para comprender y reconocer el significado de la intención comunicativa que un hablante ha emitido. El acto de escuchar implica procesos cognitivos complejos, ya que comprende la construcción de significados inmediatos. Para esto es sumamente necesario la aplicación de procesos cognitivos de construcción de significados y de interpretación de un discurso oral.

Monsalve et al. (2009) señalaron que la habilidad de lectura está relacionada con el proceso de cimentación de significados de un contenido mediante procesos complejos de coordinación e inda- 
gación que provienen de dos líneas: del mismo texto y del lector. Finalmente, en cuanto a la destreza de la escritura y la relación de textos su único propósito comunicativo es la importancia de dominar los métodos alfabéticos y los signos especiales que son atributos particulares de diferentes tipos de textos, así como las reglas lingüísticas. En cuanto al pensamiento critico Nieto y Saiz (2008), afirman que en este proceso empiezan a aparecer una serie de definiciones que caracterizarán al pensamiento crítico como de nivel superior, ya que demanda un alto uso de la reflexión, control y autorregulación que intervienen en la toma de decisiones. Paul y Elder $(2005$, p. 7$)$ indican que "el pensamiento crítico es el proceso de identificar, analizar, evaluar y argumentar. El pensamiento crítico identifica el conocimiento de las estructuras más básicas de todos estos procesos que son la clave para desarrollar el lado creativo. El verdadero pensador critico está analizando y evaluando su pensamiento constantemente"

\subsection{Habilidades del pensamiento crítico}

En la realidad en la que nos desenvolvemos es necesario conocer las habilidades que intervienen en el pensamiento crítico; en este contexto se debe desarrollar capacidades específicas que puedan potenciar lo suficiente hasta convertirse en una verdadera habilidad. Halpern (1998) hace un interesante estudio sobre las habilidades del pensamiento crítico. Según el autor, estas se dividen en cinco: la habilidad de razonamiento verbal, la habilidad de análisis de argumentos, las habilidades en el pensamiento como la prueba de hipótesis, las habilidades en el uso de riesgo e incertidumbre y, finalmente, las habilidades en la toma de decisiones y resolución de problemas.

Albertos (2015) hace una explicación de las habilidades propuestas por Halpern, en cuanto al adiestramiento del razonamiento verbal, se utiliza para vislumbrar las representaciones de persuasión que conducen al lenguaje diario, se busca explorar la relación entre lenguaje y pensamiento; el adiestramiento nos permite desempeñarnos en la vida real, aquí las explicaciones se localizan por todas partes, en la publicidad, en la dialéctica política, o en cualquier tipo de texto; en referencia a la habilidad en la prueba de hipótesis, sirve para realizar augurios automáticos sobre los acontecimientos que nos rodean, con el objetivo de dar explicación a dichos sucesos.

La habilidad en el uso de riesgo e incertidumbre juega un papel importante a la hora de asignar probabilidades a los eventos de la vida, con el propósito de tomar las mejores decisiones; en último lugar la habilidad en la toma de decisiones y la resolución de problemas involucra expresar las dificultades cabalmente e identificar los objetivos, y optar las viables opciones.

\subsection{Pensamiento crítico a través de la investigación}

Desde hace mucho tiempo se ha reflexionado sobre la importancia del pensamiento crítico, la valoración de las habilidades de este pensamiento se vincula con el conocimiento, así Uribe (2008) ha revalorado la importancia que el pensamiento crítico tiene en relación con la construcción del conocimiento ade- 
más incide en que el desarrollo del pensamiento es fundamental para generar un nuevo conocimiento.

\subsection{Dimensiones del pensamiento critico}

Valenzuela y Nieto (2007, citados en Águila, 2014) mencionan como dimensiones, la toma de decisiones, en la cual se señaló que es un proceso esencial donde se resuelven desafíos y que todos los seres humanos enfrentan en un determinado momento de su vida. Milla (2012) identificó que las opiniones deben confrontarse unas con otras en la esfera pública, y todos los participantes en este discurso público deben escuchar sinceramente los argumentos de los demás. Para tomar decisiones democráticas apropiadas, ningún grupo debe ser excluido. La dimensión resolución de problemas, se emplearía para mencionar la situación incierta en mayor o menor medida, la aplicación de instrucciones y ordenamientos por parte del solucionador se debe tener en cuenta la búsqueda acopiada en la estructura cognoscitiva.

La motivación se encuentra vinculada a diferentes aspectos como la sobrevivencia del hombre, educación, desarrollo personal, fuerza de trabajo. La motivación es un estado subjetivo de la persona como la existencia a un deseo o una necesidad. Diaz-Aguado y Baraja (1993) sostiene que la motivación esta mediada por la interacción de dos tipos de factores, los internos y los externos, los cuales permiten al individuo trazar un objetivo y buscar estrategias en función de cumplirlo; en la mayoría de los casos, la privación y la estimulación externa actúan conjuntamente para determinar la intensidad de la motivación. En lo referente a la dimensión control emocional, debería enfocarse como un recurso para gozar de mayor bienestar.

Finalmente se señala la dimensión utilidad del pensamiento Roca (2013, p. 71) sostiene que el pensamiento crítico "va más allá de las ideas de un individuo en particular, sino que está condicionado por factores emotivos, sociales, culturales, políticos, etc. que pueden apropiarse u obstaculizarlo; además remarca que es inseparable del desarrollo moral". La habilidad que tiene el individuo para pensar críticamente es importante para su vida y desarrollo personal, así como en la interrelación con los demás. Ante lo descrito el presente estudio busca determinar la relación existente entre las habilidades comunicativas y el desarrollo del pensamiento crítico en estudiantes de post grado, así como determinar la relación que existe entre la habilidad del habla, la habilidad de escucha, la habilidad de lectura, la habilidad de escritura y el perfeccionamiento que busca el pensamiento literal, inferencia y criterial.

\section{Metodología}

Hernández (2018, p. 80), menciona que los estudios descriptivos "buscan especificar las propiedades, características y los perfiles de personas, grupos, comunidades, procesos, objetos o cualquier otro fenómeno que se someta a un análisis" El objetivo de la investigación es conocer el grado de relación o e asociación que exista entre dos o más definiciones semánticas. Es un diseño no experimental no hubo manipulación de variables.

Bernal (2010, p. 250) sostiene que: "El cuestionario permite estandarizar y uniformar el proceso de recopilación de datos. Un cuestionario consiste en una serie de preguntas que responden a una o más variables que van a medirse." La muestra estuvo conformada por 40 estudiantes de postgrado, para la re- 
colección de datos se empleó como técnica la encuesta recopilando datos mediante y empleando como instrumento el cuestionario.

\section{Resultados}

Después de recolectar los datos respectivos fueron tabulados (Tabla 1) y procesados (Figura 1) obteniéndose los siguientes resultados.

Tabla 1. Niveles de las Habilidades comunicativas

$\begin{array}{lcc}\text { Niveles } & \text { Frecuencias } & \text { Porcentajes } \\ \text { Bajo } & 12 & 30 \\ \text { Medio } & 14 & 35 \\ \text { Alto } & 14 & 35 \\ \text { Total } & 40 & 100.0\end{array}$

Fuente: Elaboración propia con base en trabajo de campo.

Figura 1. Habilidades comunicativas

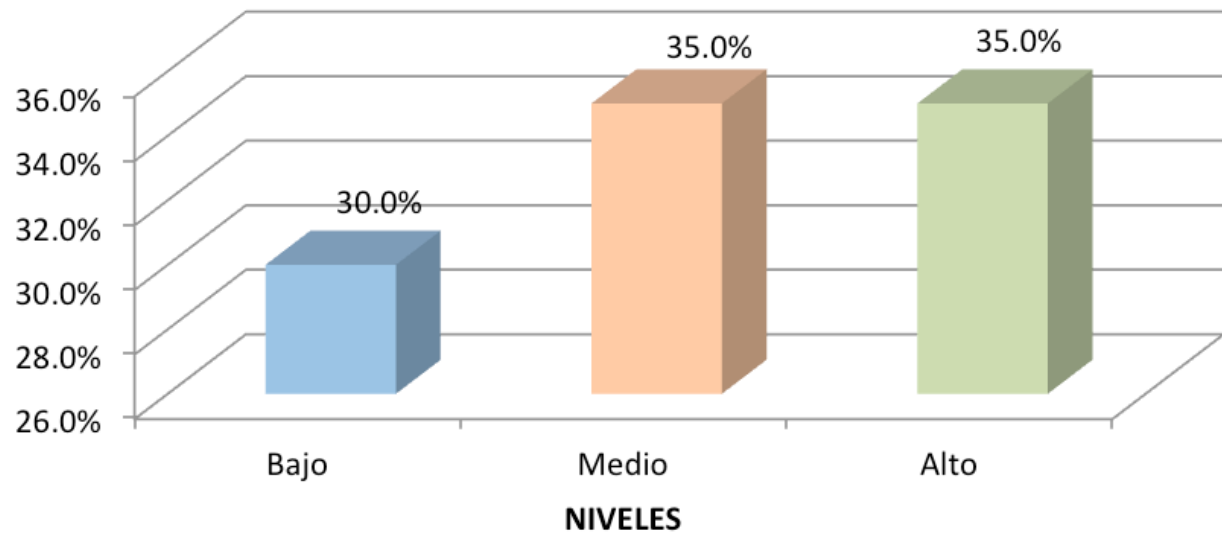

Fuente: Elaboración propia con base en trabajo de campo.

Los datos que se muestran en la Tabla 2 y Figura 2 se puede evidenciar que el $35 \%$ se ubica en un nivel alto en cuanto al desarrollo de las habilidades comunicativas, el mismo porcentaje se ubica en el nivel medio y un $30 \%$ en un nivel bajo. 
Tabla 2. Niveles del Pensamiento crítico

$\begin{array}{lcc}\text { Niveles } & \text { Frecuencias } & \text { Porcentajes } \\ \text { Bajo } & 13 & 32.5 \\ \text { Medio } & 13 & 32.5 \\ \text { Alto } & 14 & 35 \\ \text { Total } & 40 & 100.0\end{array}$

Fuente: Elaboración propia con base en trabajo de campo.

\section{Figura 2. Pensamiento crítico}

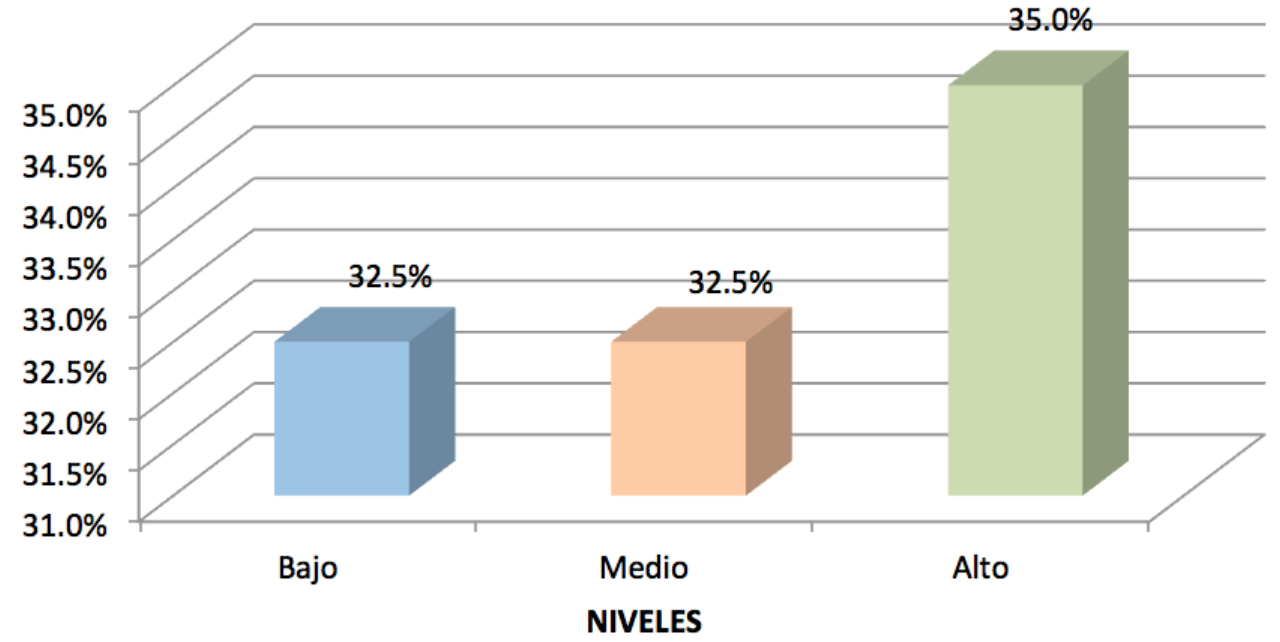

Fuente: Elaboración propia con base en trabajo de campo.

En cuanto al desarrollo del pensamiento crítico se evidencia que el 35\% de los educandos poseen un valioso $32.5 \%$ y se evidencia un nivel medio y el mismo equivalente en un nivel inferior, así se demuestra en la tabla y figura declarada.

\subsection{Proceso de contrastación de hipótesis}

En la búsqueda de la exploración investigativa se planteó hipótesis que fueron probadas con los datos recolectados obteniéndose como resultado: 
Hipótesis general

Ho: No existe una correlación positiva significativa entre las habilidades comunicativas y el desarrollo del pensamiento crítico en los estudiantes de post grado del centro de altos estudios nacionales

Hi: Existe una correlación positiva significativa entre las habilidades comunicativas y el desarrollo del pensamiento crítico en los estudiantes de post grado del centro de altos estudios nacionales.

Tabla 3. Correlación entre las habilidades comunicativas y el desarrollo del pensamiento critico

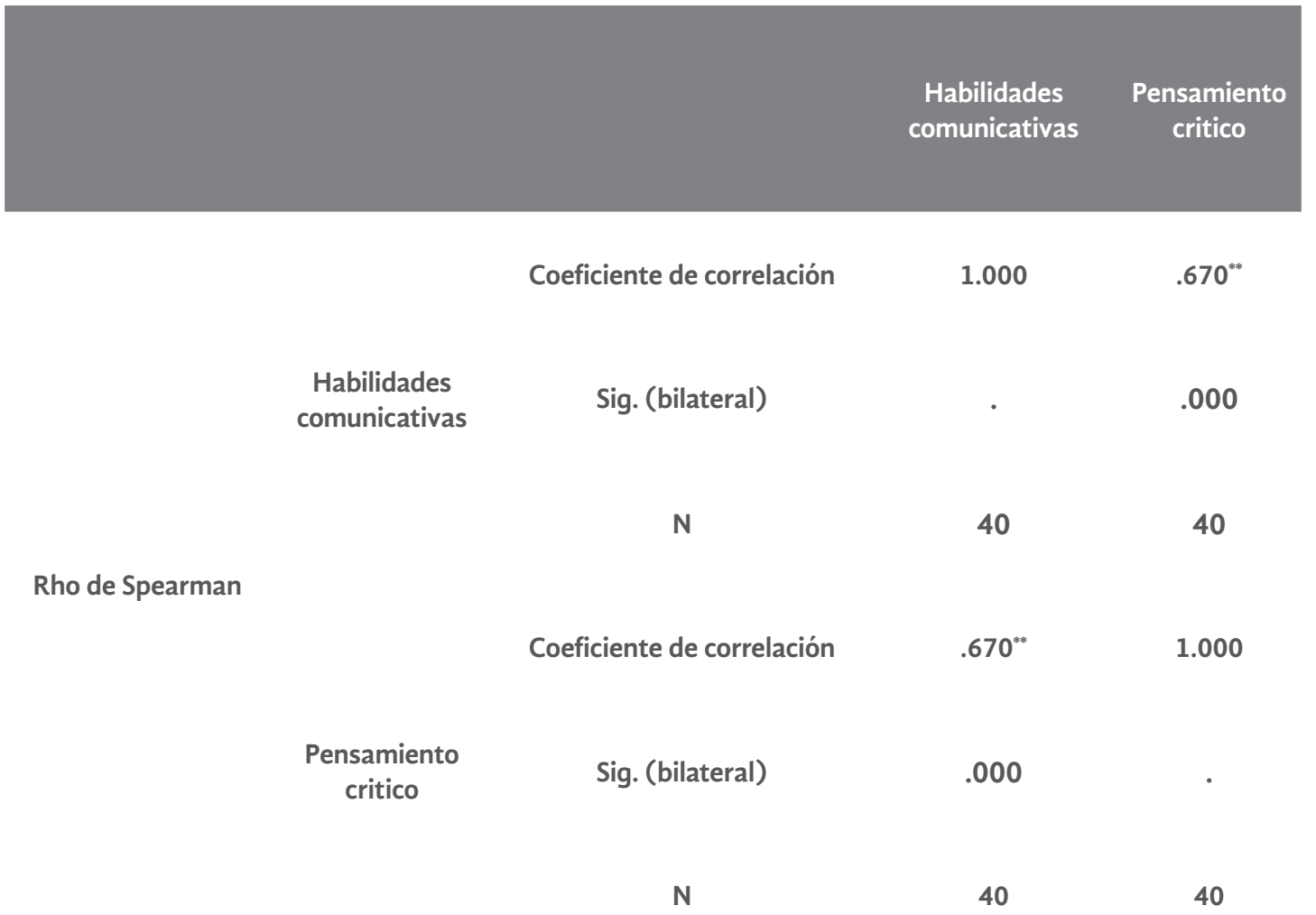

Fuente: Elaboración propia con base en trabajo de campo.

Los resultados mostrados en la tabla nos muestran que existen correlaciones significativas y positiva entre las variables en estudio $(r=0.67)$, además de obtenerse que el valor de $p=.0000$ lo cual es menor al nivel de significancia de 0.05 por lo que se puede dar por aceptada la hipótesis general de investigación.

\section{Hipótesis especifica 1}

Hi: Las habilidades del habla se relacionan positivamente con el desarrollo del pensamiento crítico en los estudiantes de post grado del centro de altos estudios nacionales. 
Tabla 4. Correlación entre las habilidades del habla y el desarrollo del pensamiento critico

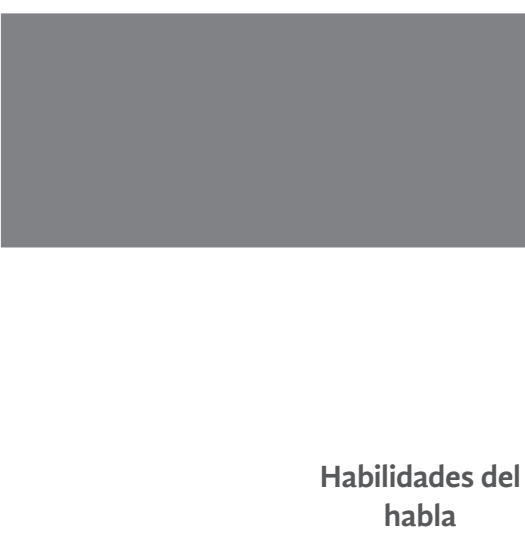

Coeficiente de correlación

1.000

$.530^{* *}$

N

40

40

Rho de Spearman

\section{Pensamiento critico}

$\mathrm{N}$
**. La correlación es significativa en el nivel 0,05 (bilateral).
Fuente: Elaboración propia con base en trabajo de campo,

Sig. (bilateral)

40

Los resultados mostrados en la tabla nos revelan que existe un grado de relación significativa entre la habilidad del habla y el desarrollo del pensamiento crítico $(\mathrm{r}=0.530)$, además de obtenerse que el valor de $\mathrm{p}=.0000$ lo cual es menor al nivel de significancia de 0.05 por lo que se puede dar por aceptada la hipótesis general de investigación.

\section{Hipótesis especifica 2}

Hi: Existe una correlación positiva significativa entre las habilidades de escucha y el desarrollo del pensamiento crítico en los estudiantes de post grado del centro de altos estudios nacionales. 
Tabla 5. Correlación entre las habilidades

de escucha y el desarrollo del pensamiento critico

Coeficiente de correlación
$\begin{aligned} & \text { Habilidades de } \\ & \text { escucha }\end{aligned}$
$\begin{aligned} & \text { Sig. (bilateral) } \\ & \text { escucha }\end{aligned}$

Los resultados presentados en la tabla nos indican que existe correlación significativa y positiva entre las habilidades de escucha y el desarrollo del pensamiento crítico $(r=0.540)$, además de obtenerse que el valor de $\mathrm{p}=.0000$ lo cual es menor al nivel de significancia de 0.05 por lo que se puede dar por aceptada la hipótesis general de investigación.

\section{Hipótesis especifica 3}

Hi: Existe una correlación positiva significativa entre las habilidades de lectura y el desarrollo del pensamiento crítico en los estudiantes de post grado del centro de altos estudios nacionales. 
Tabla 6. Correlación entre las habilidades

de lectura y el desarrollo del pensamiento critico

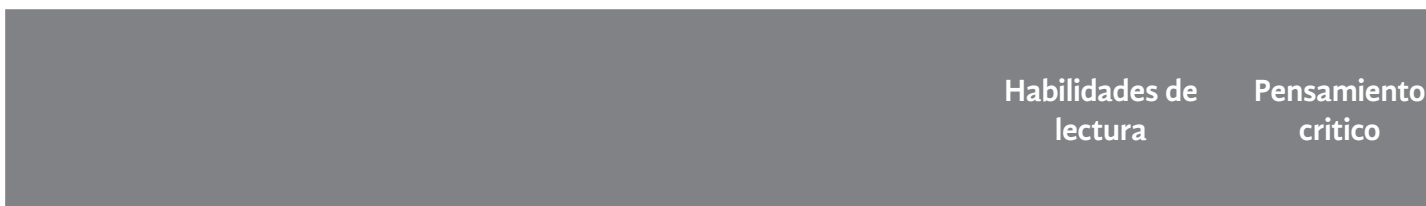

Coeficiente de correlación

1.000

$.500^{* *}$

Habilidades de lectura

N

Rho de Spearman
Sig. (bilateral)

•

.000

40

40

Coeficiente de correlación

$.500^{* *}$

1.000

Pensamiento

critico

Sig. (bilateral)

.000

N

40

40

**. La correlación es significativa en el nivel 0.05 (bilateral).

Fuente: Elaboración propia con base en trabajo de campo.

Los resultados presentados en la tabla nos indican que existe correlación significativa y positiva entre las habilidades de lectura y el desarrollo del pensamiento crítico $(r=0.500)$, además de obtenerse que el valor de $\mathrm{p}=.0000$ lo cual es menor al nivel de significancia de 0.05 por lo que se puede dar por aceptada la hipótesis general de investigación.

\section{Hipótesis especifica 4}

Hi: Existe una correlación positiva significativa entre las habilidades de escritura y el desarrollo del pensamiento crítico en los estudiantes de post grado del centro de altos estudios nacionales. 
Tabla 4. Relación entre las habilidades de escritura y el desarrollo del pensamiento critico

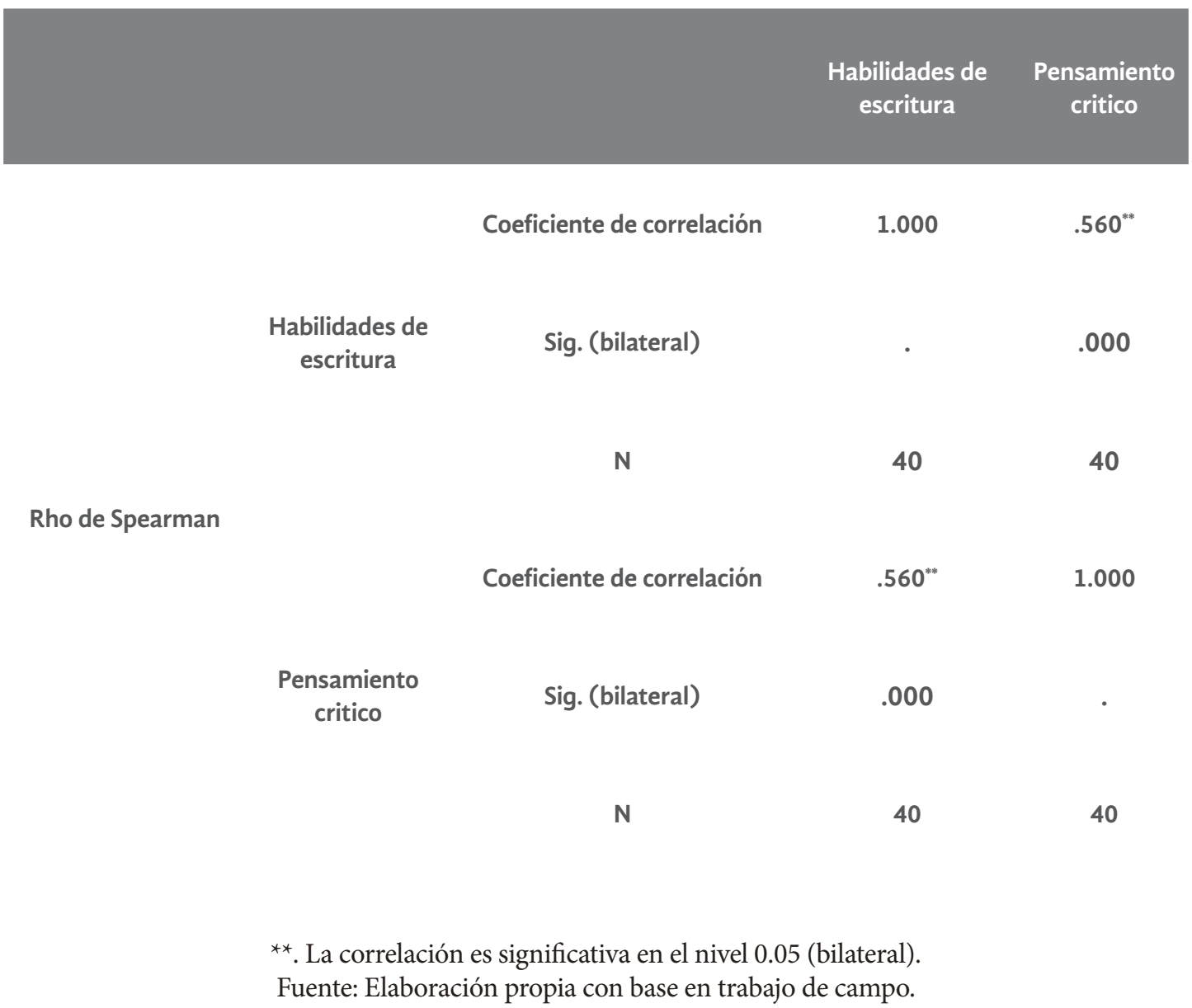

Los resultados presentados en la tabla nos indican que existe correlación significativa y positiva entre la habilidad de escritura y el desarrollo del pensamiento crítico $(r=0.560)$, además de obtenerse que el valor de $\mathrm{p}=.0000$ lo cual es menor al nivel de significancia de 0.05 por lo que se puede dar por aceptada la hipótesis general de investigación.

\section{Conclusiones}

Los resultados mostrados en las tablas permiten concluir la existencia de una correlación positiva significativa entre las habilidades comunicativas y el pensamiento crítico en estudiantes de postgrado, todo esto nos permite admitir que si mejora los niveles de habilidades comunicativas en la persona mejorará el nivel del desarrollo del pensamiento crítico. También se pudo afirmar que el 35\% está en un nivel promedio en cuanto a las habilidades comunicativas y el pensamiento crítico está en un 32.5\%. 
Los datos analizados permitieron determinar a su vez que la relación positiva entre las habilidades del escucha, habilidad del habla, habilidad de la lectura, habilidad de la escritura y el desarrollo del pensamiento crítico en los estudiantes de postgrado del centro de altos estudios nacionales, por lo cual se puede concluir que si los niveles referidos a las habilidades del habla, escucha, lectura y escritura mejora, el mismo efecto se dará en cuanto a los niveles del desarrollo del pensamiento crítico; lo mismo sucederá si disminuye en los estudiantes los niveles en cuanto a las habilidades del habla, escucha, lectura y escritura, disminuirá los niveles del desarrollo del pensamiento crítico en los estudiantes de postgrado del centro de altos estudios especializados nacionales.

\section{Referencias}

Albertos, D. (2015). Diseño, aplicación y evaluación de un programa educativo basado en la competencia científica para el desarrollo del pensamiento crítico en alumnos de educación secundaria. (Tesis doctoral). Universidad Autónoma de Madrid, España.

Águila, E. (2014). Habilidades y estrategias para el desarrollo del pensamiento crítico y creativo en alumnado de la Universidad de Sonora (Tesis de doctorado). Universidad de Extremadura, España.

Bernal, P. (2018). La Investigación en Ciencias Sociales: Técnicas de recolección de la información. Universidad Piloto de Colombia.

Cassany, D., Luna, A. y Sanz, S. (2007). Comprensión oral. En: Enseñar lengua. Barcelona: Graó.

Cortes J. (2006). El Pensamiento Crítico: Algunas reflexiones en torno a la educación contable. Ponencia presentada en $4^{\circ}$ Foro Nacional Educación Contable Universidad de Manizales Colombia.

Díaz-Aguado, M. J. y Baraja, A. (1993). Interacción educativa y desventaja sociocultural. Un modelo de intervención para favorecer la adaptación escolar en contextos interétnicos. Madrid: Centro de Investigación y Documentación Educativa.

Halpern, D. (1998). Teaching critical thinking for transfer across domains. American Psycologist, 53, 449-455. Hernández, R., Fernández, C. y Baptista, P. (2014). Metodología de la investigación. México: Mc Graw Hill. Koontz, H. y Weihrich, H. (2004). Administración, una perspectiva global (10ed.). Colombia: Mc Graw Hill Interamericana.

Lipman, M. (1997). Pensamiento complejo y educación. Madrid: Ediciones de la Torre.

Martínez, A. (2002). Comunicación Organizacional Práctica. Manual gerencial. México: Trillas.

Mendoza, P (2015) La investigación y el desarrollo de pensamiento crítico en estudiantes universitarios. (Tesis doctoral). Universidad de Málaga.

Milla, M. (2012). Pensamiento crítico en estudiantes de quinto de secundaria de los colegios de Carmen de la Legua Callao (Tesis de maestría). Universidad San Ignacio de Loyola, Lima, Perú.

Monsalve, M, Franco, M, Betancur, V, y Ramírez, D. (2009). Desarrollo de las habilidades comunicativas en la escuela nueva, Revista Educación y Pedagogía, 21(55), 189-210.

Nieto, A. M. y Saiz, C. (2008). Relación entre las habilidades y las disposiciones del pensamiento crítico. Universidad de Salamanca.

Paul, R., y Elder, L. (2003). La miniguía para el pensamiento crítico. Conceptos y herramientas. Recuperado de https://www.criticalthinking.org/resources/PDF/SP-ConceptsandTools.pdf. 
Roca, J. (2013) El desarrollo del pensamiento crítico a través de diferentes metodologías docentes en el grado de enfermería (Tesis doctoral) Universidad Autónoma de Barcelona.

Saussure, F. (1989). Curso de lingüística general. Madrid. Alianza Editorial.

UNESCO (1998). Conferencia Mundial sobre la Educación Superior. Recuperado de: http://sigc.uqroo. $\mathrm{mx} /$ Documentos\%20Externos/Educacion\%20Siglo\%20XXI\%20UNESCO.pdf.

Uribe, H. (2008). El pensamiento crítico en la educación superior. Revista Criterios, 1(1), 17-32. 